\title{
Integration of Transcriptomic And Proteomic Analyses Reveals Several Levels of Metabolic Regulation In The Excess Starch And Early Senescent Leaf Mutant Ises 1 In Rice
}

\section{Zhiming Chen}

Fujian Agriculture and Forestry University

Yongsheng Wang

Hebei University

Rongyu Huang

Xiamen University

Zesen Zhang

Xiamen University

Jinpeng Huang

Xiamen University

Yaohai Lin

Fujian Agriculture and Forestry University

Yuchun Guo

Fujian Agriculture and Forestry University

Kangjing Liang

Fujian Agriculture and Forestry University

Yuanchang Zhou

Fujian Agriculture and Forestry University

Fangyu Chen ( $\nabla$ cfy8488@aliyun.com )

Fujian Agriculture and Forestry University

\section{Research Article}

Keywords: Transcriptome, Proteome, Ises1, Leaf senescence, Transitory starch, Rice (Oryza sativa L.)

Posted Date: September 14th, 2021

DOl: https://doi.org/10.21203/rs.3.rs-805903/v1

License: (c) (1) This work is licensed under a Creative Commons Attribution 4.0 International License. Read Full License 
Page $2 / 26$ 


\section{Abstract}

Background: The normal metabolism of transitory starch in leaves plays an important role in ensuring photosynthesis, delaying senescence and maintaining high yield in crops. OsCKI1 (casein kinase I1) plays crucial regulatory roles in multiple important physiological processes, including root development, hormonal signaling and low temperature-treatment adaptive growth in rice; however, its potential role in regulating temporary starch metabolism or premature leaf senescence remains unclear. To reveal the molecular regulatory mechanism of $O s C K I 1$ in rice leaves, physiological, transcriptomic and proteomic analyses of leaves of the mutant Ises 1 (leaf starch excess and senescence 1), allelic to osck/1, and its wild-type variety (WT) were performed.

Results: Phenotypic identification and physiological measurements showed that the /ses 1 mutant exhibited starch excess in the leaves and an obvious leaf tip withering phenotype as well as high ROS and MDA contents, low chlorophyll content and protective enzyme activities compared to WT. Transcriptomic and proteomic analyses showed that the correlations of most genes at the transcription and translation levels were limited. However, the changes of several important genes related to carbohydrate metabolism and apoptosis at the mRNA and protein levels were consistent. The proteinprotein interaction (PPI) network might play accessory roles in promoting premature senescence of Ises 1 leaves. Comprehensive transcriptomic and proteomic analysis indicated that multiple key genes/proteins related to starch and sugar metabolism, apoptosis and ABA signaling exhibited significant differential expression. Abnormal increase in temporary starch was highly correlated with the expression of starch biosynthesis-related genes, which might be the main factor that causes premature leaf senescence and changes in multiple metabolic levels in leaves of Ises 1 . In addition, significant up regulation of four proteins associated with ABA accumulation and signaling were detected in the /ses 1 mutant, suggesting that ABA may involve in multiple metabolic regulation via $L S E S 1 / O S C K I 1$ and the formation of mutant phenotype in Ises 1 leaves.

Conclusion: The current study established the high correlation between the changes in physiological characteristics and mRNA and protein expression profiles in Ises 1 leaves, and emphasized the positive effect of excessive starch on accelerating premature leaf senescence. The expression patterns of genes/proteins related to starch biosynthesis and ABA signaling were analyzed via transcriptomes and proteomes, which provided a novel direction and research basis for the subsequent exploration of the regulation mechanism of temporary starch and apoptosis via $L S E S 1 / O S C K I 1$ in rice.

\section{Background}

Leaves are a critical metabolic source for plants to photosynthesize and produce organic energy materials, and the developmental status of leaves is closely related to the yield of crops. Starch, which includes storage starch in storage organs and transitory starch in photosynthetic organs, is the predominant carbohydrate synthesized by photosynthesis. It not only plays an important role in growth, development and reproduction in plants but also serves as the most important food, feed and energy 
source for human beings [1]. Therefore, the regulatory mechanism of starch synthesis, degradation and accumulation has become a research focus in plant physiology. In leaves, transitory starch is formed in chloroplasts during the day and broken down hydrolytically and phosphorolytically at night [2]. Using forward and reverse genetics, more than 40 enzymes have been discovered that are active in the process of transitory starch turnover in Arabidopsis [3]. However, the underlying mechanism with details of transitory starch turnover is presently poorly understood.

In the postgenomic era, the iTRAQ (isobaric tags for relative and absolute quantification)-based quantitative proteomics approach is an important large-scale, high-throughput analysis tool for investigating biological systems. Precise analysis of the proteome is essential for understanding the regulation of transitory starch, which needs to be further elucidated at multiple levels. Genome-wide proteomic analyses are crucial for providing accurate pictures of the regulatory networks of functional genes and proteins. However, how changes in transcriptional, posttranscriptional or even translational control are reflected in changes at the protein level remains unclear. Poor correlations between mRNA and protein expression have often been reported $[4,5]$. A recent study in a fibreless mutant of cotton using integrative transcriptome, proteome, phosphoproteome and genetic mapping emphasized the importance of the translational regulation of protein abundance [6], reflecting the significance of a comprehensive view of gene expression.

OsCKI1, which is known as $h b d 2 / L T R P K 1 / L T G 1 / E L 1$, encodes a member of the casein kinase I family and has been reported to be involved in hybrid breakdown, root development, hormone response, cold adaptation and heading [7-11], suggesting that OsCKI1 has pleiotropic functions in rice growth and development. To date, no results have linked $0 s C K / 1$ to regulating temporary starch metabolism or premature leaf senescence in rice.

Here, we report an integrative analysis of the transcriptome and proteome in an OsCKI1-deficient allelic mutant Ises 1 (leaf starch excess and senescence 1), which has an obvious premature senescence and excess starch phenotype in leaves. The physiological characteristics and differentially expressed genes/proteins in Ises 1 were analysed. The results indicated that several key functional categories, such as carbohydrate metabolism, response to hormone stimulus, pigment metabolism, translation, cytoplasmic part and plastid organization, were significantly enriched. Moreover, it was found that changes in the physiological characteristics of leaves were directly reflected in the mRNA and protein levels, which were consistent with the physiological measurement results and the omics data. Notably, this result also suggested that $A B A$ (abscisic acid) may be involved in multiple metabolic regulation via $L S E S 1 / O S C K / 1$ and the formation of mutant phenotypes in /ses 1 leaves. This result may be beneficial to the study of the genetic regulation of temporary starch in rice leaves.

\section{Methods}

\subsection{Plant materials and growing conditions}


The stably inherited mutant /ses 1 (leaf starch excess and senescence 1) with an obvious leaf tip withering phenotype was derived from the progeny of rice indica restorer line Hanhui1014 seeds treated with y-ray irradiation. Hanhui1014 plants represent the wild-type (WT). Our earlier studies have demonstrated that LSES1 encodes a CKI protein and is allelic to OSCKI1 (LOC_Os02g40860), also known as $h b d 2$ [7], LTRPK1 [8, 9], LTG1 [10] and EL 1 [11]. Similar to the mutant Itg1 [10], a T to A missense mutation at nucleotide 1070 in the coding region resulted in the amino acid substitution of isoleucine to lysine (I357K) in the LSES1 protein in mutant Ises 1 (our unpublished data). All the plant materials were provided by the Laboratory of Crop Germplasm Resources Innovation and Utilization, Fujian Agriculture and Forestry University. Rice plants were cultivated under natural conditions in a paddy field Fujian Agriculture and Forestry University. Pregerminated seeds were sown, and the seedlings were transplanted with an interplant spacing of $20 \times 20 \mathrm{~cm}^{2}$.

\subsection{Determination of starch content and $\mathrm{I}_{2}-\mathrm{Kl}$ staining in leaves}

The phenotype of excessive starch and premature senescence of Ises 1 leaves appeared from three-leaf stage to tillering stage. Therefore, the leaves of WT and Ises 1 at six-leaf stage which is the transition period between seedling stage and tillering stage were used for the following analyses. The second leaf from the top of WT and Ises 1 seedlings at the sixth leaf stage was harvested at the end of the night (6:00) and at the end of the day (18:00), frozen in liquid nitrogen and stored at $-80^{\circ} \mathrm{C}$. Approximately $0.2 \mathrm{~g}$ frozen leaf samples were ground using a mortar and pestle under cryogenic conditions. The leaf powder was measured by using a starch content assay kit (BC0700, Solarbio, China) according to the manufacturer's assay procedure. At the same time, the first, second, and third leaves from the top of the WT and Ises 1 seedlings were sampled and stained with $\mathrm{I}_{2}$-KI solution as described by Hagen et al (2008) with minor modifications [12]. The leaves were incubated in an acetone/ethanol (1:1, v/v) mixture and placed at room temperature for 24-36 h until the chlorophyll was removed. After bleaching, the leaves were put in $\mathrm{I}_{2}-\mathrm{KI}$ solution for approximately $20 \mathrm{~min}$ and then removed and placed on a table. Photographs were taken and saved with a camera. Three biological replicates of each sample were performed.

\subsection{Determination of photosynthetic pigment content}

The second leaf from the top of WT and Ises 1 seedlings at the sixth leaf stage was harvested at 8:30 on a sunny day. Measurement of the photosynthetic pigment content was performed according to the procedures of Wellburn (1994) with slight modification [13]. Briefly, approximately $0.1 \mathrm{~g}$ of cut leaves was soaked in a centrifuge tube with a $25 \mathrm{~mL}$ mixed solution of acetone/absolute ethanol (1:1, v/v). After 24 $\mathrm{h}$ in the dark, the absorbance values of the soaking liquid at wavelengths of $663 \mathrm{~nm}, 645 \mathrm{~nm}$ and $470 \mathrm{~nm}$ were measured separately by using a spectrophotometer (Beckman Coulter-DU720, USA). Then, the contents of $\mathrm{Chl} a, \mathrm{Chl} b$ and total $\mathrm{Chl}$ were calculated by referring to standard formulas. Three biological replicates of each sample were performed.

\subsection{Determination of lipid peroxidation and ROS-scavenging enzymes}


The second leaf from the top of WT and Ises 1 seedlings at the sixth leaf stage of was sampled at 8:30 on a sunny day and quickly frozen in liquid nitrogen before storage at $-80^{\circ} \mathrm{C}$. The weighed frozen leaf samples were ground on ice by using a mortar and pestle containing phosphate buffer. After grinding, the homogenate was used to determine the contents of $\mathrm{MDA}, \mathrm{H}_{2} \mathrm{O}_{2}, \mathrm{O}_{2}$ - and $\cdot \mathrm{OH}$ and the activities of CAT, SOD and POD according to the methods of kits purchased from the Nanjing Jiancheng Bioengineering Institute (China). The kits were A003-1, A064, A052, A018, A007-1, A001-1 and A084-3, respectively. Three biological replicates of each sample were performed.

\subsection{RNA-seq assays and data analysis}

Ten centimetre long segments of the leaf tip from the second leaf from the top of seedlings at the sixth leaf stage were harvested at the end of the night (6:00), quickly frozen in liquid nitrogen and stored at $-80^{\circ} \mathrm{C}$. Three biological replicates of each sample were performed, and each replicate contained ten leaf segments. RNA-seq analysis was performed by Annoroad Gene Technology (Beijing, China). Total RNA was extracted from leaves with TRIzol reagent (Invitrogen) following the manufacturer's instructions. The RNA concentration was measured with a Nanodrop spectrophotometer (Thermo), and RNA integrity was checked using a $1 \%$ agarose gel and an Agilent 2100 Bioanalyzer. After poly(A) mRNA isolation, cDNA libraries for RNA-seq were constructed using an Illumina gene expression sample prep kit (Illumina). Deep sequencing of the CDNA libraries was accomplished by Annoroad Genome. The reference genome information was from the Rice Genome Annotation Project (http://rice.plantbiology.msu.edu). The total reads of every gene locus were counted using ERANGE software (http://woldlab.caltech.edu/gitweb/). The expression level of each gene was normalized to the RPKM (reads per kilobases per million reads) value.

\subsection{Total protein extraction, digestion and iTRAQ labeling}

The leaf samples used for iTRAQ analysis were the same as those used for RNA-seq analysis. Protein extraction and digestion of each sample for proteomic analysis were performed according to Han et al (2014) with minor modifications [14]. iTRAQ labelling of peptides was performed using an iTRAQ 4-plex kit (Applied Biosystems, USA) according to the manufacturer's instructions, dried by vacuum centrifugation and stored at $-80^{\circ} \mathrm{C}$ until LC-MS/MS analysis.

\subsection{LC-MS/MS analysis}

Samples were analysed on a nanoElute (plug-in V1.1.0.27; Bruker, Bremen, Germany) coupled to a timsTOF Pro (Bruker, Bremen, Germany) equipped with a CaptiveSpray source. All raw files were analysed by Peaks Studio X software (Bioinformatics Solutions Inc., Waterloo, ON, Canada). Data were searched against the rice UniProt Reference Proteome with isoforms (downloaded December 2019). De novo sequencing of peptides, database searching and characterization of specific PTMs were performed to analyse the raw data; the false discovery rate (FDR) was set to $\leq 1 \%$, and $[-10 * \log (\mathrm{p})]$ was calculated accordingly, where $\mathrm{p}$ is the probability that an observed match is a random event. 


\subsection{Bioinformatics analysis}

The differentially expressed proteins (DEPs) and genes (DEGs) between WT and Ises 1 were identified by using the DESeq R package [15]. Proteins with a fold change greater than 1.5 were considered DEPs. The genes that had a fold change greater than 2.0 and a p-value $<0.05$ were regarded as DEGs. The hierarchical cluster analysis of DEGs according to the gene expression pattern in each sample was performed utilizing the pheatmap package in R [16]. Gene Ontology (GO) enrichment analyses of DEGs and DEPs were performed by using the BiNGO plugin in Cytoscape based on the parameter FDR $=0.05$ [17]. The protein-protein interactions in rice were assessed by the STRING database (http://string-db.org/), and the functional clusters of the protein-protein interaction network containing DEPs were distinguished by the MCODE [18] plugin in Cytoscape with built-in parameters.

\section{Results}

\subsection{Phenotypic and physiological characteristics of Ises 1 leaves}

No phenotypic differences between the Ises 1 mutant and WT were observed before the three-leaf stage. However, compared with WT plants, the older leaf blades of Ises 1 started to wither at the leaf tips from the third-leaf stage and began to exhibit a premature leaf senescence phenotype (Fig. 1-A left), and the withering degree deepened with the development process and gradually developed at higher leaf positions (Fig. 1-A mid and right). The starch contents in leaves were determined at 6:00 (the end of night, EN) and 18:00 (the end of day, ED); the values of Ises 1 at 18:00 and 6:00 were 1.9 and 2.7 times that of WT, respectively, and the value at 6:00 was slightly lower than that at 18:00 in /ses1 (Fig. 1-B left and Table S1). In the same period, seedlings of the Ises 1 mutant and WT were stained with $\mathrm{I}_{2}-\mathrm{KI}$ solution. At 18:00, the leaves of the three leaf positions in Ises 1 and WT were dyed dark blue by $\mathrm{I}_{2}-\mathrm{KI}$ solution, but the colour of Ises 1 leaves was slightly darker. At 6:00, the colour of WT leaves was pale yellowish brown, while the leaves of Ises 1 were still blue to varying degrees (Fig. 1-B right). Taken together, the results indicated that the starch produced by photosynthesis in the leaves of Ises 1 during the day was incompletely decomposed and utilized at night.

Furthermore, determinations of other physiological characteristics were also performed in the leaves of Ises 1 and WT at the seedling stage. The results showed that the contents of $\mathrm{H}_{2} \mathrm{O}_{2}, \mathrm{O}_{2}, \cdot \cdot \mathrm{OH}$ and MDA of Ises 1 leaves were significantly higher than those of WT leaves to varying degrees (Fig. 1-C top and Table S1), while the activities of SOD, POD and CAT were significantly lower in Ises1 than in WT (Fig. 1-C bottom-left and Table S1). The $\mathrm{Chl} a, \mathrm{Chl} b$ and total Chl contents in Ises 1 leaves were all significantly lower than those in WT leaves (Fig. 1-C bottom-right and Table S1). These results implied that increased lipid peroxidation, an imbalance of ROS-scavenging systems and chlorophyll degradation occurred in Ises 1 leaves.

\subsection{Identification and analysis of DEGs}


To obtain more information at the mRNA level, a comparative transcriptomic analysis of Ises 1 and WT leaves was performed. The quality of the sequencing results showed that after the transcriptome sequencing data of each sample were filtered based on the clean Q30 base rate (\%), the proportion of bases with a quality value greater than 30 (error rate less than $0.1 \%$ ) in the total set exceeded $90 \%$ (Fig. 2A and Table S2), indicated that these results were sufficient for subsequent analyses. In this study, 4989 (2883 upregulated and 2106 downregulated) differentially expressed genes (DEGs) were identified between Ises 1 and WT with the following parameters: $p$-value $<0.05$ and fold change $>2$ (Fig. 2-B and Table S3). As shown in Fig. 2-C, these DEGs were congregated into two distinct modules by Hierarchical Clustering according to the expression level of each gene in the samples. Gene Ontology enrichment analyses of biological process (GO-BP) for DEGs were performed, and the top 15 functional subcategories were selected to create a bubble chart. As shown in Fig. 2-D, several key processes, such as carbohydrate metabolic process, oxidation reduction, starch metabolic process, response to abiotic stimulus, nucleotide metabolic process, alcohol metabolic process and pigment metabolic process, were significantly enriched.

\subsection{Identification and analysis of DEPs}

Proteome profile analyses were performed on the leaves of Ises 1 and WT. A total of 82903 peptides were detected, and the lengths of peptides, as determined by proteomics, ranged mostly from 6 to 12 aa (Fig. 3-A and Table S4). A total of 3192 proteins containing at least two unique peptides were detected from these peptides, and 568 differentially expressed proteins (DEPs), including 455 upregulated proteins and 113 downregulated proteins, were identified between Ises 1 and WT (Fig. 3-B and Table S4). GO enrichment analysis of these 568 DEPs was performed, and the top 10 functional subcategories of each of the three $\mathrm{GO}$ categories were selected to create a bar graph. As shown in Fig. 3-C, in the biological process category, translation was the most enriched subclass, followed by cellular biosynthetic process, biosynthetic process, and monosaccharide metabolic process. In the cellular component category, cytoplasmic part was the subclass with the highest enrichment score, followed by cytoplasm, cytosolic part, and ribosome. In the molecular function category, structural molecule activity occupied the top place, followed by structural constituent of the ribosome, rRNA binding, and RNA binding.

\subsection{Functional clusters in the PPI network}

To explore the biological characteristics of the 568 differentially expressed proteins, a protein-protein interaction (PPI) network was constructed by integrating these proteins and the known STRING interactions and distinguishing the functional clusters via the MCODE [18] plugin for Cytoscape according to the default parameters. In total, four highly related functional clusters were identified and named clusters 1, 2, 3 and 4, which included 50, 16, 14 and 25 proteins, respectively (Fig. 4-A and Table S5). Notably, several proteins related to carbohydrate metabolism were found to interact such as glucose6-phosphate 1-dehydrogenase and ATP-dependent 6-phosphofructokinase. Moreover, a Gene Ontology enrichment analysis of biological process (GO-BP) for these four clusters was performed, and the top 10 functional subcategories of each cluster were selected to create a bar graph. As shown in Fig. 4-B, several 
key processes were significantly enriched in the four clusters: protein metabolic process, macromolecule metabolic process, photosynthesis, plastid organization, catabolic process, hexose metabolic process, lipid metabolic process and organic acid process. Overall, the PPI network containing these four functional clusters might play an accessory role in the formation of excess starch and premature aging phenotype in Ises 1 leaves.

\subsection{Carbohydrate metabolism- and apoptosis metabolism-associated genes/proteins}

Thus far, comprehensive proteome and transcriptome studies have shown that the correlation between mRNA levels and protein expression across large data sets is generally weak $[4,5,19]$. To more fully clarify the regulation mechanism of temporary starch and premature senescence of rice leaves, the differentially expressed genes/proteins related to carbohydrate metabolism and apoptosis metabolism were comprehensively screened from WT and Ises 1 in the following analysis.

More than 240 genes/proteins involved in carbohydrate metabolism were identified between /ses 1 and WT (Table S6). As expected, several carbohydrate metabolism-related genes in Ises 1 showed a consistent trend of changes at the mRNA and protein levels. For example, the genes encoding glucose-1-phosphate adenylyltransferase large subunit (AGP-L), chloroplastic maltose excess protein 1-like (MEX1), soluble starch synthase 1 (SSS1), sucrose synthase 1 (SUS1), fructokinase 2 (FRK-2), pyrophosphate-fructose 6phosphate 1-phosphotransferase subunit alpha (PFP-ALPHA), cytosolic pyruvate kinase isozyme (PK), malate dehydrogenase (MDH), alcohol dehydrogenase 1 (ADH1) and trehalose-6phosphate synthase 3 (TPS3) all exhibited increased transcripts and protein abundance (Table S6). Other genes/proteins in Ises 1, such as the genes encoding chloroplastic $\beta$-amylase 3 (BAM3) involved in starch metabolism, glyceraldehyde-3-phosphate dehydrogenase (GAPC) involved in glycolysis and trehalase (TRE) involved in trehalose metabolism also showed altered transcription levels (Table S6). Overall, the up- and down-regulation of these genes/proteins might lead to the imbalance of carbohydrate metabolism in Ises 1 leaves.

Furthermore, more than 50 genes/proteins involved in apoptosis-related metabolism in Ises 1 showed increased or decreased abundance (Table S7). Notably, the genes encoding L-ascorbate peroxidase (APX1; APX3) involved in ROS scavenging, nonspecific lipid-transfer protein (nsLTP2) involved in phospholipid metabolism, and adenine phosphoribosyltransferase form 2 (APRT) involved in nucleic acid metabolism were regulated at both mRNA and protein levels (Table S7). Several genes/proteins associated with ROS production, such as the protein photosystem I iron-sulfur centre (psaC) and the gene encoding cytochrome bc1 complex subunit (CR7) in Ises 1 exhibited increased protein and transcript abundance, respectively (Table S7). Besides, chlorophyll metabolism-associated genes, such as the genes encoding chloroplastic protein STAY-GREEN (SGR), putative red chlorophyll catabolite reductase ( $\mathrm{rccR}$ ) and $\mathrm{ABC}$ transporter $\mathrm{C}$ (ABCC3) in /ses 1 also showed higher transcription levels than those in WT (Table S7). Taken together, the transcription or abundance of these genes/proteins increased and decreased, revealing changes in chlorophyll metabolism and redox 
homeostasis in Ises 1 leaves, which was basically consistent with the previous physiological characteristic detection results.

\subsection{Multiple metabolic regulation mediated by differentially abundant genes/proteins}

A possible schematic for the metabolic regulation mediated by partial differentially abundant genes/proteins was provided to explain the changes in multiple metabolic levels in the leaves of /ses 1 . As shown in Fig. 5 and Table S8, 35 genes were used to describe the pathways related to carbohydrate metabolism, redox homeostasis and cell signaling in Ises 1 leaves, and the changes in transcription and protein levels of each gene were marked. The results implied that the excessive accumulation of tempoary starch might be the main factor of premature senescence and imbalance of carbohydrate metabolism in /ses 1 leaves, and the mutant gene LSES1/OsCKI1 may regulate starch metabolism and cell apoptosis through ABA-mediated signaling.

\section{Discussion}

\subsection{Premature senescence and excess starch phenotype induced by LSES1 mutation}

Assimilate partitioning has long been recognized as a target for crop improvement because it can limit the yield potential of crop plants. A phenotype involving an excessive accumulation of starch and growth retardation is often observed when any inhibition in the export of photoassimilate or in starch metabolism occurs in the leaves [20]. In this study, the OsCKI1-deficient allelic mutant Ises 1 showed obvious premature senescence and an excess starch phenotype in leaves. Compared with WT, older leaf blades in Ises 1 displayed chlorosis at the tip from the third-leaf stage, and the chlorosis degree deepened with the development process and gradually developed to higher leaf positions (Fig. 1-A). In particular, the starch content and $\mathrm{I}_{2}-\mathrm{KI}$ staining analysis indicated that $/$ ses 1 presented an abnormal starch accumulation phenotype in leaves during premature senescence (Fig. 1-B and Table S1). This phenotype was similar to that of three reported rice mutants, es/10 [21], ossac3 [22] and ossac4 [23]. Although LSES1 is allelic to $O s C K I 1 / h b d 2 / L T R P K 1 / L T G 1$ and the mutation site in the /ses 1 mutant is similar to that in the Itg1 mutant (our unpublished data), no results have been reported on the premature senescence and excess starch phenotype in the leaves of OsCKI1-deficient allelic mutants except Ises 1 . Therefore, the Ises 1 mutant is an ideal material for studying the molecular mechanism of premature leaf senescence and temporary starch metabolism via LSES1/OSCKI1 and the pleiotropism of LSES1/OSCKI1 in rice.

\subsection{Global changes in the transcriptome and proteome in /ses1}

To comprehensively explore the mechanism of premature leaf senescence caused by temporary starch regulation and the changes in metabolic levels during senescence, transcriptome and proteome analyses were conducted, and the results showed global changes in mRNA and protein levels in Ises 1 leaves (Fig. 2-C and Fig. 3-B). Furthermore, subsequent functional analysis of the 4989 differentially expressed genes (DEGs) and 568 differentially expressed proteins (DEPs) showed that the DEGs were mainly involved in carbohydrate metabolic process, oxidation reduction and response to abiotic stimulus (Fig. 2-D), while the 
DEPs were heavily involved in translation, cytoplasmic part and biosynthetic process (Fig. 3-D). Gene expression is usually affected by transcription, posttranscriptional regulation, RNA splicing, translation, and posttranslational modification, which results in changes in mRNA and protein levels that are not always the same. In this study, approximately $20 \%$ of the identified proteins were also found to be regulated at the transcriptional level. The congruence between proteomics and transcriptome data seems to be very poor, which is consistent with general observations $[4,5,18,24,25]$.

\subsection{Metabolic imbalance induced an excessive accumulation of starch and affected glycolysis/TCA cycle in Ises 1 leaves}

The metabolism of temporary starch may be a complex network formed by the coordinated expression of numerous genes. In this study, several key proteins involved in starch biosynthesis, such as AGPL (glucose-1-phosphate adenylyltransferase large subunit), GBSSII (granule-bound starch synthase II), SSS1 (chloroplastic soluble starch synthase 1) and SSI (chloroplastic starch synthase I), all exhibited increased abundance in Ises 1 (Fig. 5). Notably, AGP-L is the first rate-limiting enzyme in starch biosynthesis, and its overexpression rice plants show significantly increased starch content in leaves [26]. Moreover, several $\beta$-amylases in Ises 1 showed down-regulated transcription levels. A striking sample is BAM3 (Fig. 5), which encodes the most important $\beta$-amylase that strongly participates in the decomposition of temporary starch in leaves at night, and its functional deletion mutant exhibits a higher transitory starch accumulation phenotype in leaves than does other $\beta$-amylase deletion mutants [27]. Excessive accumulation of leaf starch affects the normal development of plants, resulting in plant growth retardation, decreased yield, and even premature senescence, which has been reported in typical model plants, such as rice and Arabidopsis [21, 28, 29].

Furthermore, the expression levels of multiple genes/proteins related to carbohydrate metabolism downstream of starch metabolism in Ises 1 were almost all upregulated (Fig. 5). SUS (sucrose synthase 1) overexpression provides more UDP-glucose and fructose for various metabolic pathways [30], while FRK (fructokinase-2) upregulation catalyses the formation of more fructose-6-phosphate derived from fructose and channels sucrose into the glycolytic pathway [31]. It could be speculated that the substrate of glycolysis is more biased towards fructose and sucrose rather than glucose derived from starch due to the accumulation of starch in the chloroplast, which reduces the transport of maltose and glucose to the cytoplasm. The increased intermediate products further induce the upregulation of downstream glycolysis/TCA cycle-related genes (Fig. 5). On the other hand, the additional sucrose consumption seemed to induce the upregulation of MEX1 (chloroplastic maltose excess protein 1-like) and TRE (trehalase) to maintain a stable sucrose content (Fig. 5). MEX1 is the main carrier of maltose from chloroplasts to the cytoplasm for sucrose synthesis [32], while TRE is involved in trehalose metabolism, playing an important role in regulating the balance of sucrose and trehalose contents [33].

Taken together, these results implied that the metabolic imbalance of transitory starch, which appeared as increased starch biosynthesis and incomplete starch degradation, induced the excessive accumulation 
of starch in Ises 1 leaves, which coincided with the abovementioned phenotype and physiological characteristics in Ises1.

\subsection{Increased premature senescence-related metabolism in Ises1}

Comparative transcriptomic and proteomic analyses implied that in addition to carbohydrate metabolism-related genes/proteins, other mechanisms might contribute to explaining the premature senescence phenotype of Ises 1 mutant leaves. The leaves of Ises 1 presented increased ROS and MDA contents compared to WT (Fig. 1-C top). These physiological differences were also reflected in the changes in mRNA and protein levels. For example, the protein psaC (photosystem I iron-sulfur centre) and gene CR7 (cytochrome bc1 complex subunit, complex III) in /ses 1 showed increased protein and transcript abundance, respectively (Fig. 5). Among them, psaC is involved in ROS generation in the chloroplastic electron transport chain [34], while CR7 affects the electron transfer rate of the mitochondrial respiratory chain [35]. The leaves of Ises 1 showed lower SOD, CAT and POD activities compared with WT (Fig. 1-C bottom-left). Interestingly, several L-ascorbate peroxidases, such as APX1 and APX3, in Ises 1 all exhibited increased transcripts and decreased protein abundance (Fig. 5), which suggested that the expression of APXs is posttranscriptionally regulated [36]. In addition, the contents of chlorophyll a, chlorophyll b and total photosynthetic pigment in Ises 1 leaves were significantly higher than those in WT (Fig. 1-C bottom-right). As expected, several photosynthetic pigment anabolismassociated genes in Ises 1 were regulated. For example, CAO (chlorophyllide a oxygenase), which is involved in chlorophyll $b$ biosynthesis [37], and rccR (putative red chlorophyll catabolite reductase), which is involved in chlorophyll a degradation [38], showed down- and upregulated transcription levels, respectively (Fig. 5). Notably, SGR in Ises 1 exhibited reduced transcription (Fig. 5); this gene triggers chlorophyll degradation in natural or dark-induced leaf senescence [39], and both its deletion and overexpression will affect the degradation of chlorophyll in rice leaves [40].

In brief, the results showed increased premature senescence-related metabolism in Ises 1 and suggested consistency between the transcriptomic and proteomic analyses and the physiological determination of lipid peroxidation and ROS-scavenging enzymes.

\subsection{Increased nucleotide degradation and inhibited cell proliferation in Ises1}

Senescence is considered a self-saving strategy in plants, during which plants recycle and deliver nutrients from degraded proteins, lipids, and nucleic acids to still growing sites [41]. A similar senescenceinduced plant adaptation also occurred in the Ises 1 mutant described herein. In this study, APRT (adenine phosphoribosyltransferase form 2) in Ises 1 showed a higher protein abundance level than that in WT (Fig. 5); this gene is involved in step 1 of the subpathway in the salvage pathway that synthesizes AMP (adenosine monophosphate) from degraded adenine [42]. Interestingly, TET6 (tetraspanin-6), which is considered a senescence-associated protein and is involved in leaf and root growth via negative regulation of cell proliferation in Arabidopsis [43], was found to be expressed in Ises 1 but not in WT (Fig.

5). Additionally, KRP6 (cyclin-dependent kinase inhibitor 6) was found to be overexpressed in /ses 1 mutant (Fig. 5), which accumulates in Arabidopsis lacking CK1s/AELs will inhibit cell proliferation [44]. 
Taken together, these results implied that increased RNA/DNA degradation (a trait of PCD), nutrient recycling of nucleotides and inhibited cell proliferation occurred in the Ises 1 mutant during senescence.

\subsection{ABA may involve in multiple metabolic regulation via $L S E S 1 /$ OsCKI1}

The rapid accumulation of $A B A$ is considered one of the key characteristics of plants in response to abiotic stress [45], and it is related to the changes in starch metabolism induced by stress [46]. In this study, four proteins associated with ABA accumulation and signaling, including ASR5 (abscisic stressripening protein 5), ASR2 (abscisic stress-ripening protein 2 fragment), PYL5 (ABA receptor 5) and SAPK (serine/threonine-protein kinase), showed significantly increased abundances in the /ses1 mutant (Fig. 5). Among them, ASR5 is involved in the ABA-mediated stomatal closure pathway in response to drought and osmotic stress, and rice plants overexpressing ASR5 gene exhibit higher endogenous ABA accumulation [47]. PYL5 functions as a positive regulator of abiotic stress-responsive gene expression [48], which together with SAPK2, is part of an ABA signaling unit that modulates seed germination and early seedling growth [49]. OsCKI1/hbd2/LTRPK1/LTG1/EL1, which is involved in hybrid breakdown, root development, hormone response, cold adaptation and heading, has been cloned utilizing multiple rice mutants with different phenotypes [7-11]. Notably, Liu et al. (2003) showed that OsCKI1 may be involved in the regulation of gene expression and mediating the interaction of $A B A$ and other hormones signaling through hierarchical phosphorylation [8]. Recent study reports that mutation of the CK1s causes reduced phosphorylation, ubiquitination, and degradation of ABA receptors PYR/PYLs, thereby enhancing the ABA responses [50], which is similar to the result observed in the Ises 1 mutant. In addition, transcriptional and translational control seems to play important roles in the regulation of starch metabolism in response to stress. Multiple primary carbohydrate metabolism-related genes were found to be regulated by ABA in previous transcriptomic and proteomic studies [51-53]. For example, AGPL (glucose-1-phosphate adenylyltransferase large subunit) which encodes the large subunits of AGPase involving in starch biosynthesis showed up-regulated expression in Ises 1 mutants (Fig. 5). The accumulation of AGP-Ls transcript is known to cooperatively regulated by ABA and sucrose, and their expression patterns were elevated significantly by the co-treatment of sucrose and $A B A[54,55]$. Taken together, these results implied that $L S E S 1 / O S C K I 1$ mutation might enhance the ABA response, and then the increased $A B A$ cooperated with sucrose to regulate downstream starch metabolism-related gene expression, which ultimately leads to excessive starch and premature senescence phenotypes in the leaves of Ises 1 mutant.

\subsection{The PPI network may affect the development and metabolism of leaves in Ises1}

Analysing protein-protein interaction (PPI) networks has become increasingly important for further exploring the biological characteristics of proteins during plant development. In this study, 568 DEPs were used to form a PPI network, and four highly related functional clusters were found in this interactome by the MCODE [18] Cytoscape plugin (Fig. 4-A). In cluster 1, four proteins, A2XZF9, B8AZD7, B8ACZ5 and B8AE20, encoding the eukaryotic translation initiation factor 3 subunit, were found to interact, which may be due to their functional links in modulating the global translation rate to regulate cell growth, 
proliferation and differentiation [56]. These four proteins all showed upregulation in Ises 1 (Table S4). In cluster 2, B8AGW7 (acetyltransferase component of pyruvate dehydrogenase complex) interacted with A2XTX6 (3-oxoacyl-[acyl-carrier-protein] synthase). The pyruvate dehydrogenase complex synthesizes acetyl-CoA, which binds to acyl carrier proteins to participate in fatty acid biosynthesis [57]. Both B8AGW7 and A2XTX6 also showed upregulation in Ises 1 (Table S4). In cluster 3, two carbohydrate metabolism-related proteins were found to interact: A2WLL3 (ATP-dependent 6-phosphofructokinase) and A2YKG1 (glucose-6-phosphate 1-dehydrogenase). Their interaction is due to the connection of substrates between glycolysis and the pentose phosphate pathway. Both A2YKG1 and A2WLL3 were upregulated in Ises 1 (Table S4). In cluster 4, two proteins related to phospholipid metabolism were found to interact: B8AL07 (phosphatidylserine decarboxylase proenzyme 1) and Q9LKM2 (phospholipase D). Their interaction also includes $\mathrm{N}$-acylphosphatidylethanolamines (NAPEs), which exist during cell damage [58]. Phosphatidylserine decarboxylase proenzyme 1 and phospholipase $D$ are involved in the synthesis and decomposition of NAPEs, respectively $[59,60]$. Interestingly, Q9LKM2 was downregulated but B8AL07 was upregulated in Ises 1 (Table S4), which suggested that these two proteins may have an antagonistic effect. Taken together, these results implied that the PPI network might play an accessory role in promoting the apoptosis-related metabolism level of Ises 1 leaves.

\section{Conclusion}

The osck/1 allelic mutant /ses1, which displayed premature senescence and an excess starch phenotype, was comprehensively analysed with transcriptomic and proteomic approaches. The differentially expressed genes (DEGs) and proteins (DEPs) revealed changes in several important metabolic mechanisms, such as carbohydrate metabolism, pigment metabolism, redox regulation and cell signaling. A possible schematic for the metabolic regulation mediated by differentially abundant genes/proteins was provided to explain the changes in multiple metabolic levels in the leaves of Ises 1 (Fig. 5 and Table S8). The results implied that the excessive accumulation of temporary starch in leaves was the main factor that induced premature senescence of Ises 1 leaves and changes in several carbohydrate metabolism levels (including glycolysis/TCA cycle and trehalose metabolism) downstream of starch metabolism. The protein-protein interaction (PPI) network might play an auxiliary role in promoting premature leaf senescence in Ises1. In addition, the possible potential roles of the mutant LSES1/OsCKI1 gene in cell signaling were discussed. The analysis results suggested that $L S E S 1 / O S C K I 1$ might participate in the regulation of starch metabolism, cell apoptosis and ABA-mediated signaling, but future studies are required to verify it. Altogether, our study provides novel insight into the metabolic mechanism of temporary starch via $L S E S 1 / O S C K I 1$ in rice leaves.

\section{Abbreviations}

ABA: Abscisic acid; AMP: Adenosine monophosphate; CAT: Catalase; Chl: Chlorophyll; DEGs: Differentially expressed genes; DEPs: Differentially expressed proteins; ED: End of day; EN: End of night; 
FDR: False Discovery Rate; GO: Gene Ontology; GO-BP: Gene ontology of biological process; iTRAQ: Isobaric tags for relative and absolute quantification; MDA: Malondialdehyde; MT: Mutant; NAPEs: Nacylphosphatidylethanolamines; PCD: Programmed cell death; POD: Peroxidase; PPI: Protein-protein interaction; PTMs: Posttranslational modifications; ROS: Reactive oxygen species; SOD: Superoxide dismutase; WT: Wild-type variety.

\section{Declarations}

\section{Ethics approval and consent to participate}

Not applicable.

\section{Consent for publication}

Not applicable.

\section{Availability of data and materials}

All data generated or analysed during this study are included in this published article and its supplementary information files. The sequencing data associated with transcription profiles in this study have been deposited in NCBI SRA database with accession number PRJNA757395 (https://www.ncbi.nlm.nih.gov/bioproject/PRJNA757395).

\section{Competing interests}

The authors declare that they have no competing interests.

\section{Funding}

This work was supported by Natural Science Foundation of Fujian Province (2019J01376) and grants from the Seed Industry Innovation and Industrialization Project of Fujian Province (zycxny2021004).

\section{Authors' contributions}

FYC and YCZ conceived and designed the study. ZMC, YSW, RYH, ZSZ, and JPH performed the experiments. ZMC, YSW and YHL analyzed high-throughput sequencing data. ZMC, YCG, KJL and FYC analyzed the data as a whole and wrote the manuscript. All authors read and approved the final manuscript.

\section{Acknowledgements}

The authors are grateful to Dr. Changchuan Xie and Dr. Yaying Wu (Analysis and Testing Center, School of Life Sciences, Xiamen University) for their help in mass spectrometry analysis. 


\section{References}

1. Geigenberger P. Regulation of starch biosynthesis in response to a fluctuating enviorenment. Plant Physiol. 2011; 155(4): 1566-77.

2. Lloyd JR, Kossmann J. Transitory and storage starch metabolism: two sides of the same coin? Curr Opin Biotech. 2015; 32: 143-48.

3. Mahlow S, Orzechowsk S, Fettke J. Starch phosphorylation: insights and perspectives. Cell Mol Life Sci. 2016; 73(14): 2753-64.

4. Greenbaum D, Colangelo C, Williams K, Gerstein M. Comparing protein abundance and mRNA expression levels on a genomic scale. Genome Biol. 2003; 4(9): 4007-21.

5. Xiong Q, Feng J, Li ST, Zhang GY, Qiao ZX, Chen Z, Wu Y, Lin Y, Li T, Ge F, Zhao JD. Integrated transcriptomic and proteomic analysis of the global response of Synechococcus to high light stress. Mol Cell Proteomics. 2015; 14(4): 1038-53.

6. Ma QF, Wu CH, Wu M, Pei WF, Li XL,Wang WK, Zhang JF, Yu JW, Yu SX. Integrative transcriptome, proteome, phosphoproteome and genetic mapping reveals new aspects in a fiberless mutant of cotton. Scientific Rep-uk. 2016; 6(1): 1088-97.

7. Yamamoto E, Takashi T, Morinaka Y, Lin SY, Wu JZ, Matsumoto T, Kitano H, Matsuoka M, Ashikari M. Gain of deleterious function causes an autoimmune response and Bateson-DobzhanskyMuller incompatibility in rice. Mol Genet Genomics. 2010; 283(4): 305-15.

8. Liu W, Xu ZH, Luo D, Xue HW. Roles of OsCKI 1, a rice casein kinase I, in root development and plant hormone sensitivity. Plant J. 2003; 36(2): 189-202.

9. Liu W, Ji SX, Fang XL, Wang QG, Li Z, Yao FY, Hou L, Dai SJ. Protein kinase LTRPK1 influences cold adaptation and microtubule stability in rice. J Plant Growth Regul. 2013; 32: 483-90.

10. Lu GW, Wu FQ, Wu WX, Wang HJ, Zheng XM, Zhang YH, Chen XL, Zhou K N, Jin MN, Cheng ZJ, Li $X Y$, Jiang L, Wang HY, Wan JM. Rice LTG1 is involved in adaptive growth and fitness under low ambient temperature. Plant J. 2014; 78(3): 468-80.

11. Dai C, Xue HW. Rice early flowering1, a CKI, phosphorylates DELLA protein SLR1 to negatively regulate gibberellin signalling. EMBO J. 2010; 29(11): 1916-27.

12. Hagen SR, Muneta P, Tourneau DL, Brown J. Effect of temperature on the starch content of potato callus tissue. Am J Potato Res. 2008; 68(3): 191-5.

13. Wellburn AR. The spectral determination of chlorophylls a and $b$ as well as total carotenoids using various solvents with spectrophotometers of different resolution. Plant Physiol. 1994; 144(3): 30713.

14. Han C, He DL, Li M, Yang PF. In-depth proteomic analysis of rice embryo reveals its important roles in seed germination. Plant Cell Physiol. 2014; 55(10): 1826-47.

15. Anders S, Huber W. Differential expression analysis for sequence count data. Genome Biol. 2010; 11(21): 1344-9. 
16. Guan D H, Tian HL. Integrated network analysis to explore the key genes regulated by parathyroid hormone receptor 1 in osteosarcoma. World J Surg Onc. 2017; 15(1): 177-87.

17. Maere S, Heymans K, Kuiper M. BiNGO: a Cytoscape plugin to assess overrepresentation of gene ontology categories in biological networks. BMC Bioinformatics. 2005; 21(16): 3448-9.

18. Bader GD, Hogue CW. An automated method for finding molecular complexes in large protein interaction networks. BMC Bioinformatics. 2003; 4(1): 2.

19. Soto-Suárez M, Serrato AJ, Rojas-González JA, Bautista R, Sahrawy M. Transcriptomic and proteomic approach to identify differentially expressed genes and proteins in Arabidopsis thaliana mutants lacking chloroplastic 1 and cytosolic FBPases reveals several levels of metabolic regulation. BMC Plant Biol. 2016; 16(1): 258-24.

20. Hirose T, Aoki N, Harada Y, Masaki O, Yoichi H, Ryu O, Miyao A, Hirohiko H, Tomio T. Disruption of a rice gene for a-glucan water dikinase, OSGWD1, leads to hyperaccumulation of starch in leaves but exhibits limited effects on growth. Front Plant Sci. 2013; 4: 147-56.

21. Chen XL, Zhu MD, Gu FX, Liu MM, Zhang YY, Xing YD, Du D, Xiao YH, Zhu XY, He GH. Identification and gene fine mapping of starch accumulation and early senescent leaf mutant esl10 in rice. Crop Sci. 2018; 58(1): 204-17.

22. Huang JY, Yan M, Zhu XY, Zhang T, Shen WQ, Yu P, Wang YT, Sang XC, Yu GL, Zhao BB, He GH. Gene mapping of starch accumulation and premature leaf senescence in the ossac3 mutant of rice. Euphytica. 2018; 214(10):1-14.

23. Zhu MD, Chen XL, Zhu XY, Xing YD, Du D, Zhang YY, Liu MM, Zhang QL, Lu X, Peng SS, He GH, Zhang TQ. Identification and gene mapping of the starch accumulation and premature leaf senescence mutant ossac4 in rice. J Integr Agr. 2020; 19(9): 2150-64.

24. Gygi SP, Rochon Y, Franza BR, Aebersold R. Correlation between protein and mRNA abundance in yeast. Mol Cell Biol. 1999; 19(3): 1720-30.

25. Pandey A, Mann M. Proteomics to study genes and genomes. Nature. 2000; 405(6788): 837-46.

26. Oiestad AJ, Martin JM, Giroux MJ. Yield increases resulting from AGPase overexpression in rice are reliant on plant nutritional status. Plant Growth Regul. 2019; 89(2), 179-90.

27. Fulton DC, Stettler M, Mettler T, Vaughan CK, Li J, Francisco P, Gil M, Reinhold H, Eicke S, Messerli G, Dorken G, Halliday K, Smith AM, Smith SM, Zeeman SC. Beta-amylase4, a noncatalytic protein required for starch breakdown, acts upstream of three active beta-amylases in Arabidopsis chloroplasts. Plant Cell. 2008; 20(4): 1040-58.

28. Weise SE, Aung K, Jarou ZJ, Mehrshahi P, Li Z, Hardy AC, Carr DJ, Sharkey TD. Engineering starch accumulation by manipulation of phosphate metabolism of starch. Plant Biotechnol J. 2012; 10(5): 545-54.

29. Stitt M, Zeeman SC. Starch turnover: pathways, regulation and role in growth. Curr Opin Plant Biol. 2012; 15(3): 282-92.

30. Wang AY, Man-Han K, Yang WH, Sayion Y, Liu LF, Lee PD, Su JC. Differentially and developmentally regulated expression of three rice sucrose synthase genes. Plant Cell Physiol. 1999; 40(8): 800-7. 
31. Šimon M, Shen ZJ, Ghoto K, Chen J, Liu X, Gao GF, Kokalj AJ, Novak S, Drašler B, Zhang JY, You YP, Drobne D, Zheng HL. Proteomic investigation of Zn-challenged rice roots reveals adverse effects and root physiological adaptation. Plant Soil. 2020; 460: 69-88.

32. Niittylä T, Messerli G, Trevisan M, Chen J, Smith AM, Zeeman SC.

A previously unknown maltose transporter essential for starch degradation in leaves. Science. 2004; 303(5654): 87-9.

33. Müller J, Aeschbacher RA, Wingler A, Boller T, Wiemken A. Trehalose and trehalase in Arabidopsis. Plant Physiol. 2001; 125(2):1086-93.

34. Kozi A. The water-water cycle in chloroplasts: scavenging of active oxygens and dissipation of excess photons. Annu Rev Plant Phys. 1999; 50(1): 601-39.

35. Zeng DY, Cui J, Yin YS, Zang M, Shan S, Gao X, Zhang YC, Sun YQ, Lu WH. Effects of space flight on expression of key proteins in rice leaves. Rice Sci. 2020; 27(5): 423-41.

36. Yoshimura K, Yabuta Y, Tamoi M, Ishikawa T, Shigeoka S. Alternatively spliced mRNA variants of chloroplast ascorbate peroxidase isoenzymes in spinach leaves. Biochem J. 1999; 338 (Pt 1): 41-8.

37. Oster U, Tanaka R, Tanaka A, Rüdiger W. Cloning and functional expression of the gene encoding the key enzyme for chlorophyll b biosynthesis (CAO) from Arabidopsis thaliana. Plant J. 2000; 21(3): 305-10.

38. Rodoni S, Vicentini F, Schellenberg M, Matile P, Hortensteiner S. Partial purification and characterization of red chlorophyll catabolite reductase, a stroma protein involved in chlorophyll breakdown. Plant Physiol. 1997; 115(2): 677-82.

39. Sakuraba Y, Schelbert S, Park SY, Han SH, Lee BD, Andrès CB, Kessler F, Hörtensteiner S, Paek NC. STAY-GREEN and chlorophyll catabolic enzymes interact at light-harvesting complex II for chlorophyll detoxification during leaf senescence in Arabidopsis. Plant Cell. 2012; 24(2): 507-18.

40. Jiang HW, Li MR, Liang NT, Yan HB, Wei YB, Xu XL, Liu J, Xu ZF, Chen F, Wu GJ. Molecular cloning and function analysis of the stay green gene in rice. Plant J. 2007; 52(2): 197-209.

41. Nir S, María MRW, Kamolchanok U, Eduardo B. Stress-induced senescence and plant tolerance to abiotic stress. J Exp Bot. 2018; 69(4): 845-53.

42. Xing QH, Ru ZG, Li J, Zhou CJ, Jin DM, Sun Y, Wang B. Cloning a second form of adenine phosphoribosyl transferase gene (TaAPT2) from wheat and analysis of its association with thermosensitive genic male sterility (TGMS). Plant Sci. 2005; 169(1): 37-45.

43. Wang F, Muto A, Van de Velde J. Functional analysis of the Arabidopsis TETRASPANIN gene family in plant growth and development. Plant Physiol. 2015; 169(3): 2200-14.

44. Li Q, Zhuang W, Chen HH, Liu T, Liao K, Xue HW. Plant casein kinases phosphorylate and destabilize a cyclin-dependent kinase inhibitor to promote cell division. Plant Physiol. 2021; kiab284.

45. Christmann A, Moes D, Himmelbach A, Yang Y, Tang Y, Grill E. Integration of abscisic acid signalling into plant responses. Plant Biol. 2006; 8(3): 314-25. 
46. Kempa S, Krasensky J, Dal Santo S, Kopka J, Jonak C. A central role of abscisic acid in stressregulated carbohydrate metabolism. PLoS One. 2008; 3: e3935.

47. Li J, Li Y, Yin Z, Jiang JH, Zhang MH, Guo X, Ye JZ, Zhao Y, Xiong HY, Zhang ZY, Shao YZ, Jiang CH, Zhang HL, An G, Paek NC, Ali J, Li ZC. OsASR5 enhances drought tolerance through a stomatal closure pathway associated with $\mathrm{ABA}$ and $\mathrm{H}_{2} \mathrm{O}_{2}$ signalling in rice. Plant Biotechnol J. 2017; 15(2): 183-96.

48. Kim H, Lee K, Hwang H, Bhatnagar N, Kim DY, Yoon IS, Byun MO, Kim ST, Jung KH, Kim BG. Overexpression of PYL5 in rice enhances drought tolerance, inhibits growth, and modulates gene expression. J Exp Bot. 2014; 65(2):453-64.

49. Kim H, Hwang H, Hong JW, Lee YN, Ahn IP, Yoon IS, Yoo SD, Lee S, Lee SC, Kim BG. A rice orthologue of the ABA receptor, OsPYL/RCAR5, is a positive regulator of the ABA signal transduction pathway in seed germination and early seedling growth. J Exp Bot. 2012; 63(2): 1013-1024.

50. Chen HH, Li Q, Xu ZH, Zhu JK, Xue HW. EL1-like casein kinases suppress ABA signaling and responses by phosphorylating and destabilizing the ABA receptors PYR/PYLs in Arabidopsis. Mol Plant. 2018;11(5):706-19.

51. Choudhury A, Lahiri A. Comparative analysis of abscisic acid-regulated transcriptomes in Arabidopsis. Plant Biol. 2011; 13(1): 28-35.

52. Huang YQ, Cai SG, Zeng JB, Wu DZ, Zhang GP. Isobaric tags for relative and absolute quantitation proteomic analysis of germinating barley under gibberellin and abscisic acid treatments. J Agric Food Chem. 2017; 65(10): 2248-57.

53. Yoshida T, Fujita Y, Maruyama K, Mogami J, Todaka D, Shinozaki K, Yamaguchi-Shinozaki K. Four Arabidopsis AREB/ABF transcription factors function predominantly in gene expression downstream of SnRK2 kinases in abscisic acid signalling in response to osmotic stress. Plant Cell Enviro. 2014; 38(1): 35-49.

54. Akihiro T, Mizuno K, Fujimura T. Gene expression of ADP-glucose pyrophosphorylase and starch contents in rice cultured cells are cooperatively regulated by sucrose and ABA. Plant Cell Physiol. 2010; 46(6): 937-46.

55. Li YP, Yu GW, Lv YA, Long TD, Li P, Hu YF, Liu HM, Zhang JJ, Liu YH, Li WC, Huang YB. Combinatorial interaction of two adjacent cis-active promoter regions mediates the synergistic induction of Bt2 gene by sucrose and ABA in maize endosperm. Plant sci. 2018; 274: 332-40.

56. Raabe K, Honys D, Michailidis C. The role of eukaryotic initiation factor 3 in plant translation regulation. Plant Physiol Biochem. 2019; 145: 75-83.

57. Magnuson K, Jackowski S, Rock CO, Cronan JE. Regulation of fatty acid biosynthesis in escherichia coli. Microbiol Rev. 1993; 57(3): 522-42.

58. Lafrance CP, Blochet JE, Pézolet M. N-acylphosphatidylethanolamines: effect of the N-acyl chain length on its orientation. Biophys J. 1997; 72(6): 2559-68.

59. Nerlich A, Orlow von M, Rontein D, Hanson AD, Dörmann P. Deficiency in phosphatidylserine decarboxylase activity in the psd1 psd2 psd3 triple mutant of Arabidopsis affects 
phosphatidylethanolamine accumulation in mitochondria. Plant Physiol. 2007; 144(2): 904-14.

60. Kilaru A, Chapman KD. N-Acylated phospholipid metabolism and seedling growth: insights from lipidomics studies in Arabidopsis. Plant Signal Behav. 2012; 7(9): 1200-2.

Figures 
Figure 1

A
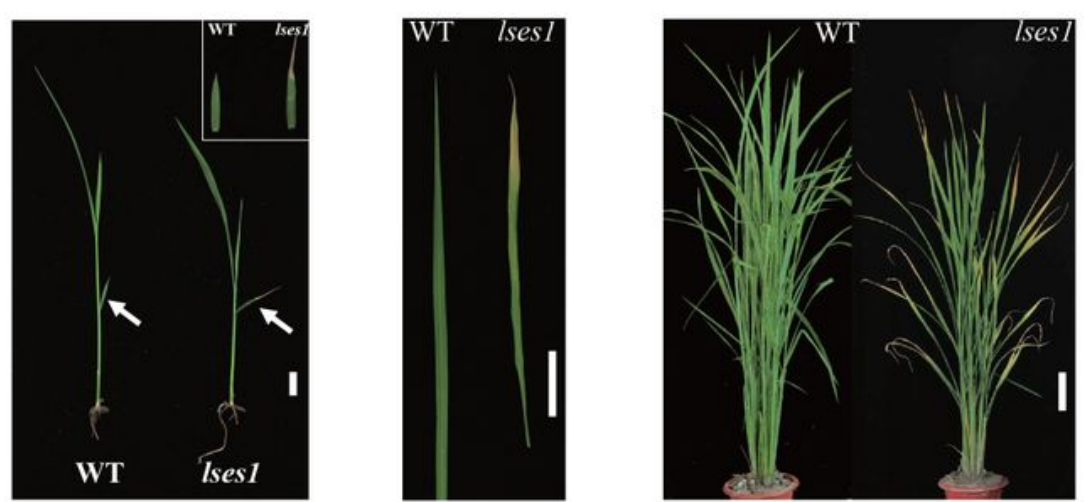

B
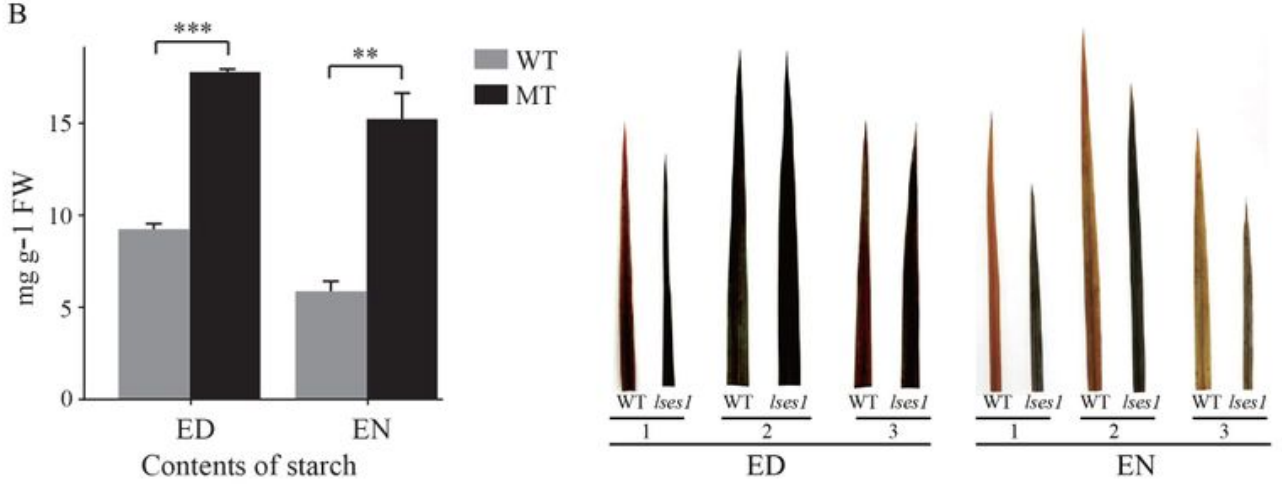

C
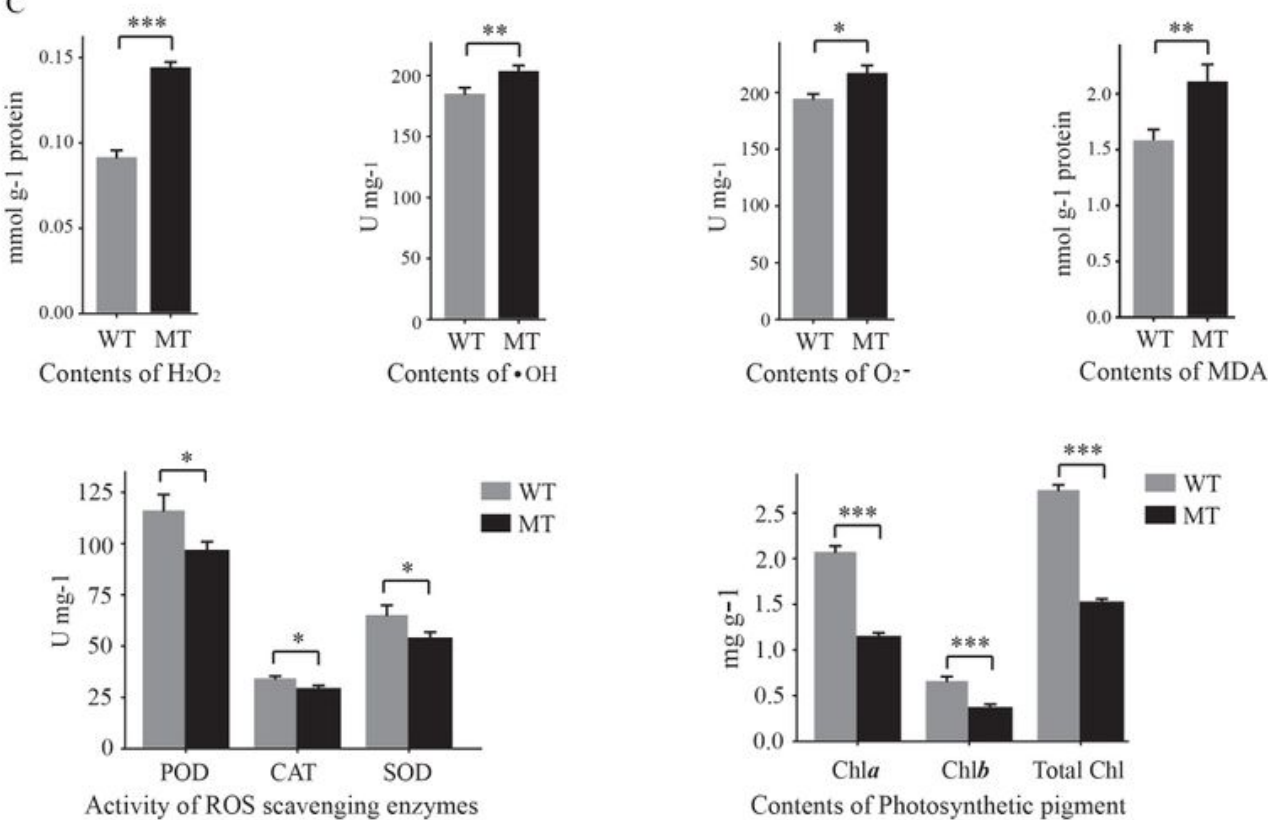

Figure 1

Phenotypes and physiological characteristics of the wild type (WT) and Ises 1 mutant. A, Phenotypes of the plants and leaves of WT and Ises 1 at the different growth stages. Phenotypes of the plants of WT and Ises 1 at the third-leaf stage (left), scale bar is $1 \mathrm{~cm}$; Phenotypes of the second leaves from the top of WT and Ises 1 seedlings at the sixth-leaf stage (mid), scale bar is $5 \mathrm{~cm}$; Phenotypes of the plants of WT and Ises 1 at the tillering stage (right), scale bar is $10 \mathrm{~cm}$. B, Starch contents (left) and I2-KI staining (right) 
of the leaves of WT and Ises1. ED, sampling at the end of the day; EN, sampling at the end of the night. 1, 2 and 3 represent the first, second and third leaves from the top of seedlings at the sixth leaf stage, respectively. C, Contents of ROS and chlorophyll and activities of protective enzymes in the leaves of wildtype (WT) and Ises 1 mutant (MT) plants. Error bars represent the standard deviations of three biological replicates. *, ** and ${ }^{* * *}$ significant difference at $\mathrm{P}<0.05, \mathrm{P}<0.01$ and $\mathrm{P}<0.001$, respectively.

Figure 2

A

Filter Distribution

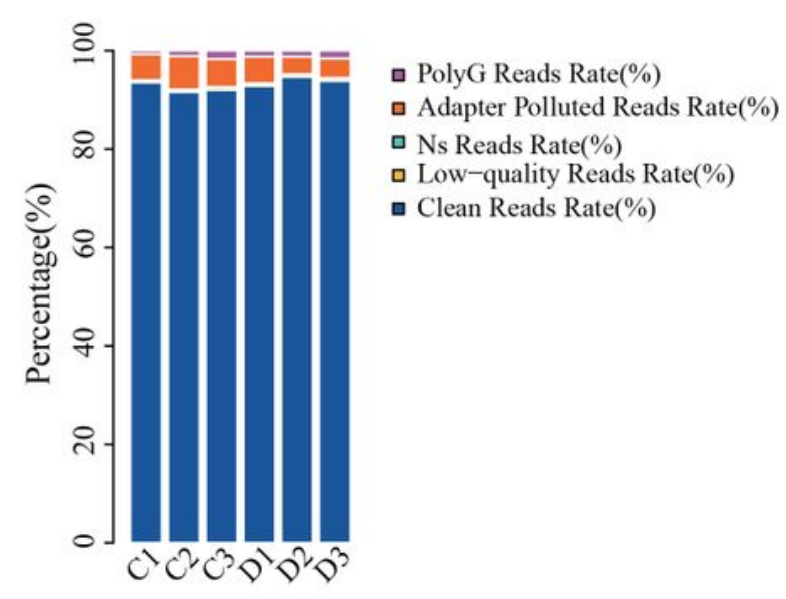

B

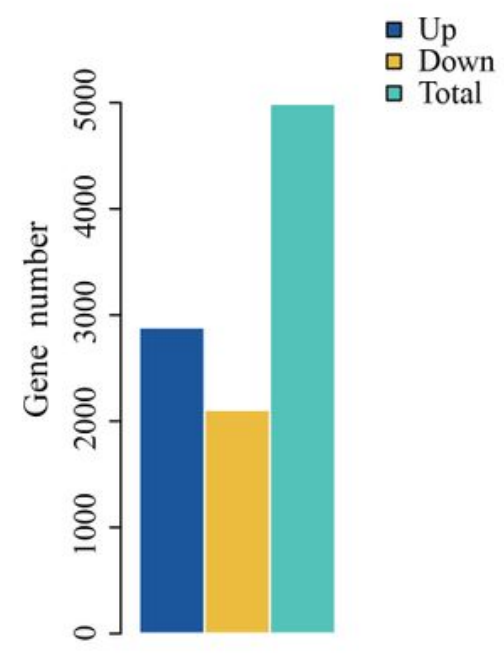

$\mathrm{C}$

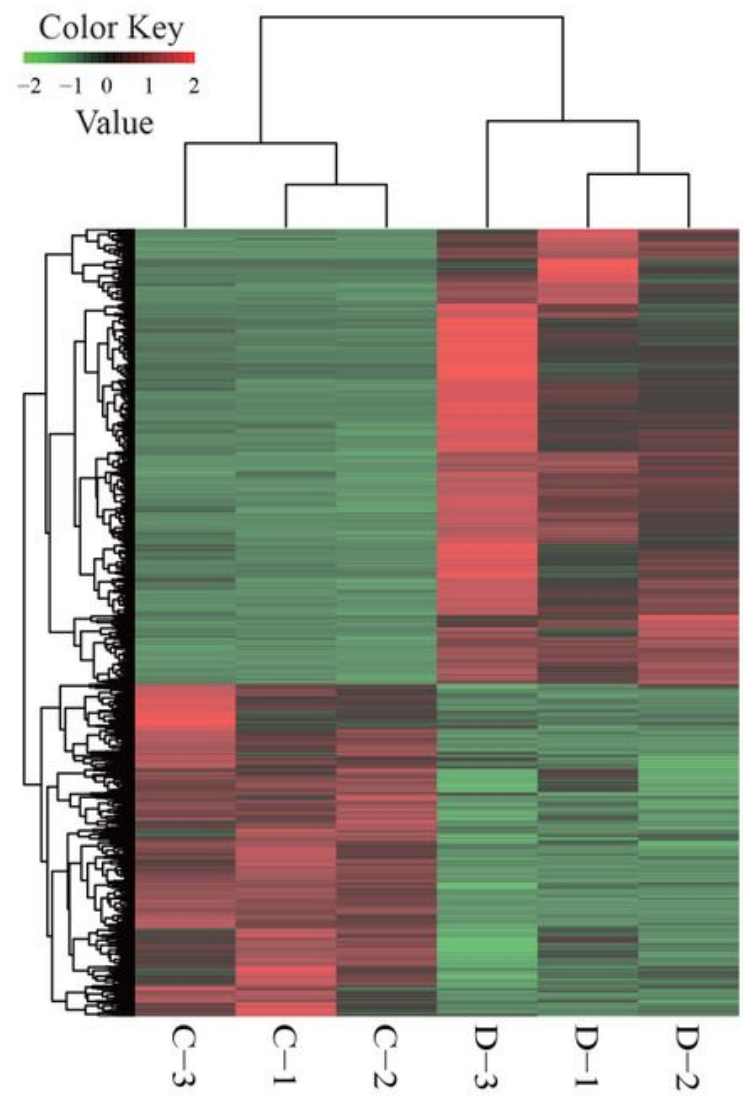

$\mathrm{D}$

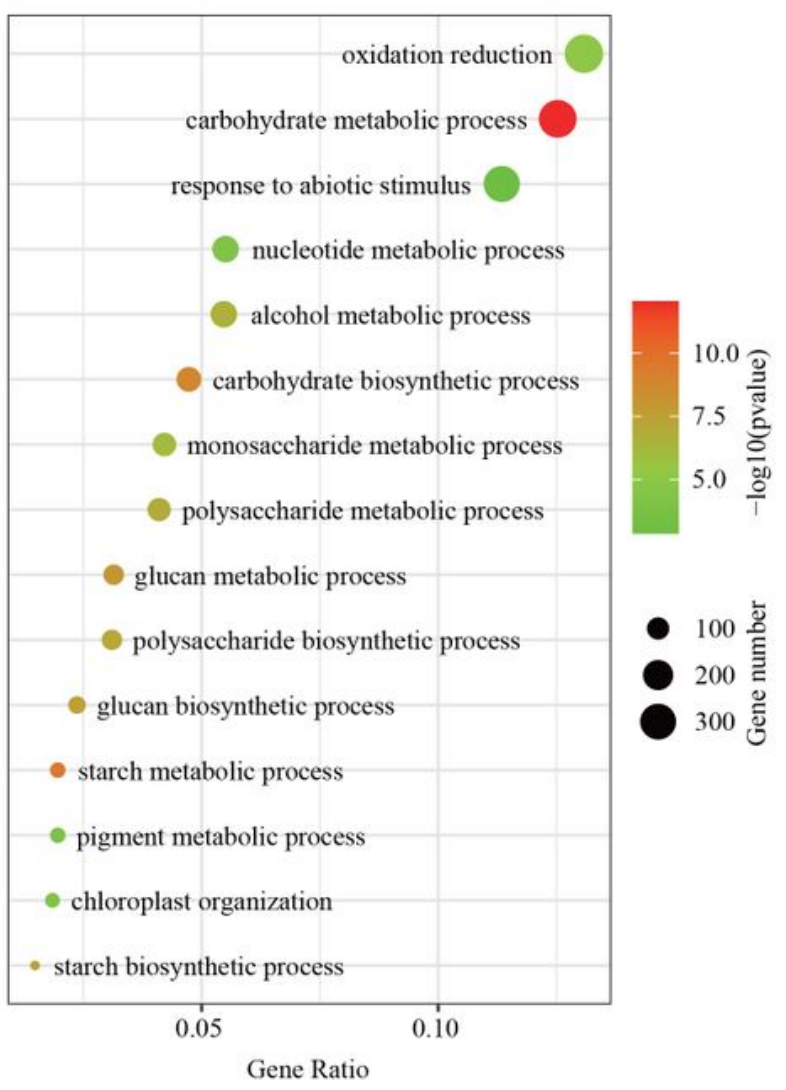

Figure 2 
Identification and analysis of DEGs (differentially expressed genes) expressed in clusters between the wild type (WT) and the Ises 1 mutant. A, The ratio of reads in the transcriptome. C1-C3, wild-type repeats 1-3; D1-D3, Ises1 mutant repeats 1-3. B, Statistical results of the DEGs. C, Heatmap of the DEGs. C1-C3, wild-type repeats 1-3; D1-D3, Ises1 mutant repeats 1-3; I and II represent clusters I and II with similar gene expression patterns, respectively. D, GO-BP enrichment analysis of DEGs from the two clusters. The top $15 \mathrm{GO}$ categories of each cluster are shown.

Figure 3
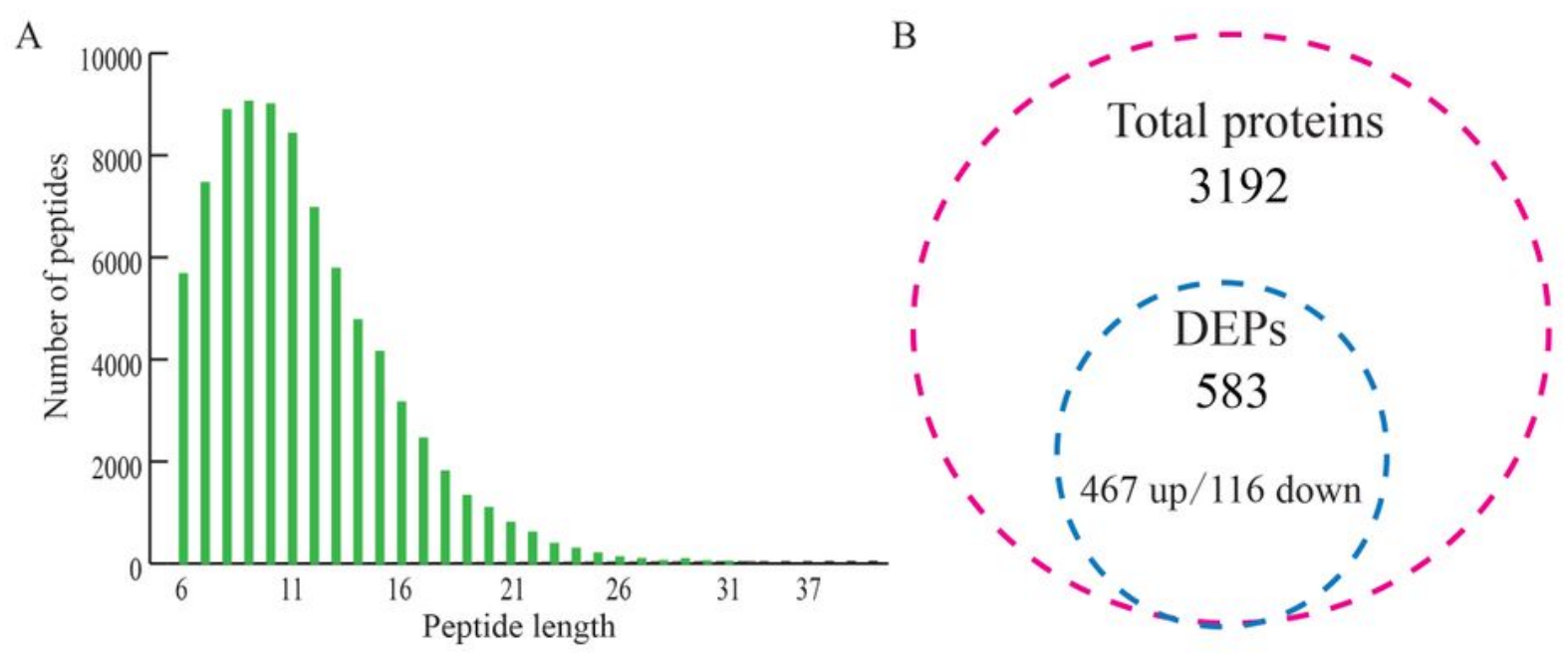

$\mathrm{C}$
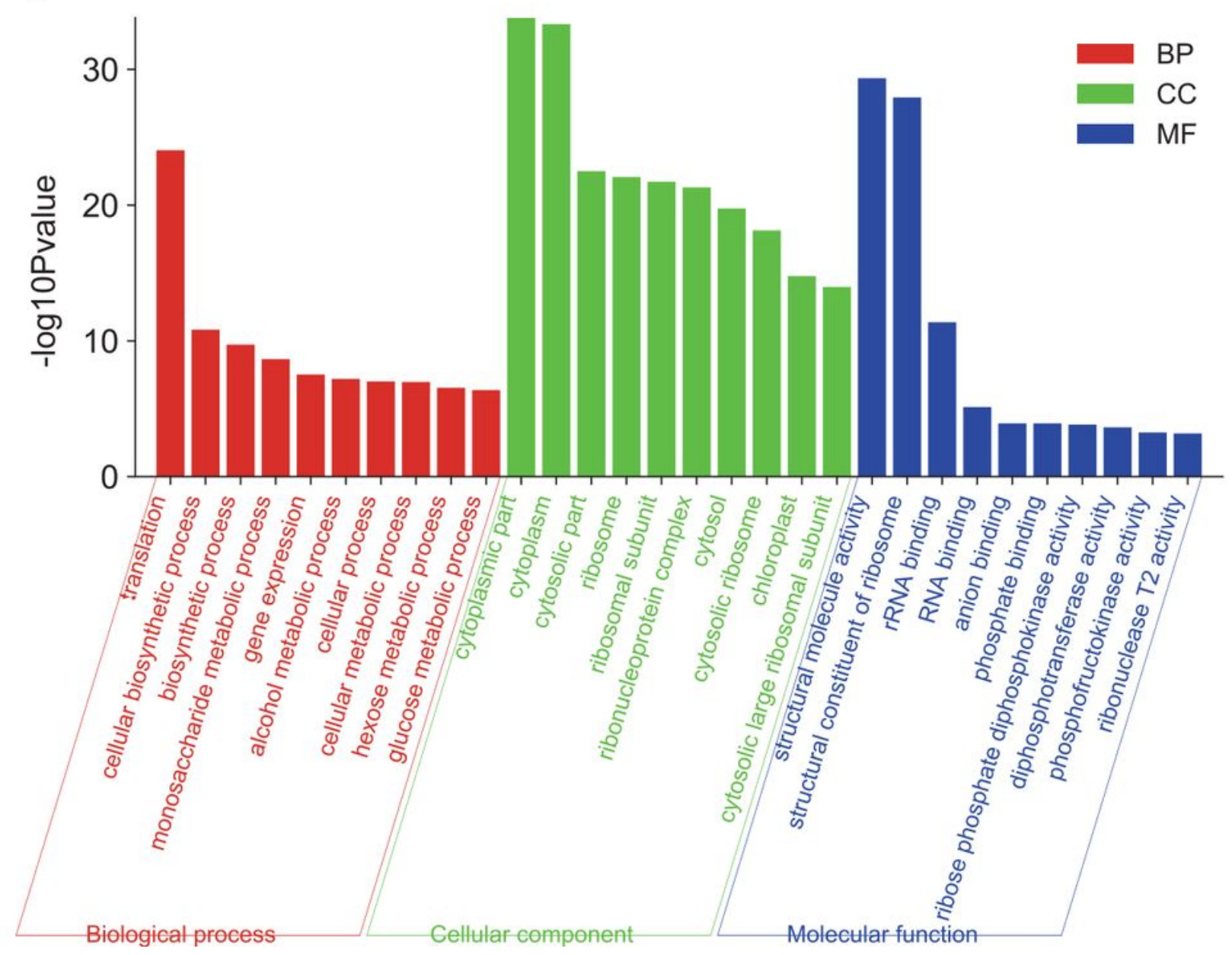

Figure 3 
Identification and analysis of DEPs (differentially expressed proteins) between the wild type (WT) and the Ises 1 mutant. A, Distribution of peptide length and number in the whole proteome. B, Statistical results of the DEPs. C, GO enrichment analysis of the DEPs. The top $10 \mathrm{GO}$ functional subcategories of the three main categories are shown.

Figure 4

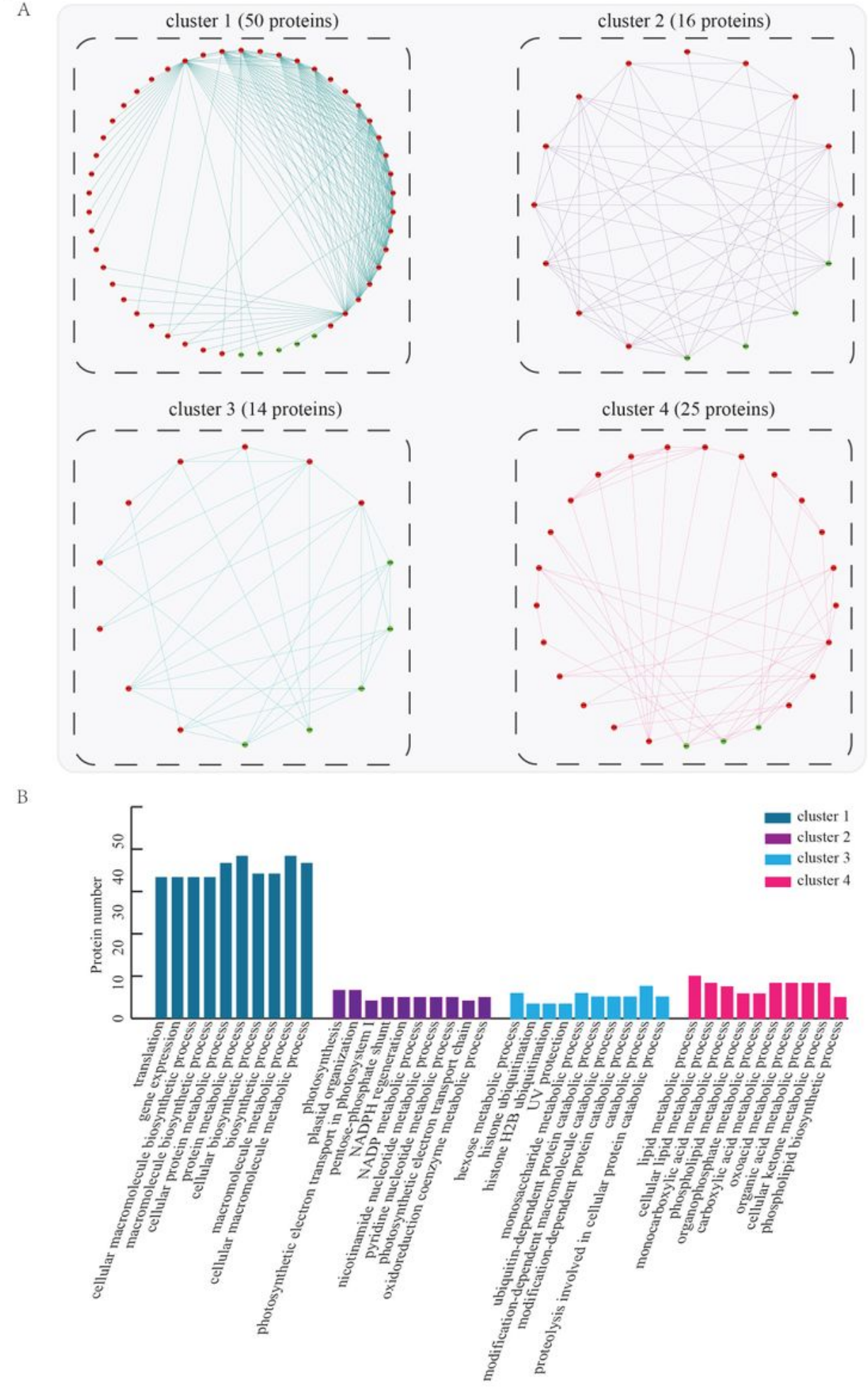

Figure 4 
Functional cluster analyses of the PPI (protein-protein interaction) network. A, Four functional clusters distinguished from the PPI network. Red dots represent upregulated proteins, and green dots represent downregulated proteins. B, GO-BP enrichment analyses of proteins in each of 4 clusters. The bar graph shows the top $10 \mathrm{GO}$ categories in each cluster.

Figure 5

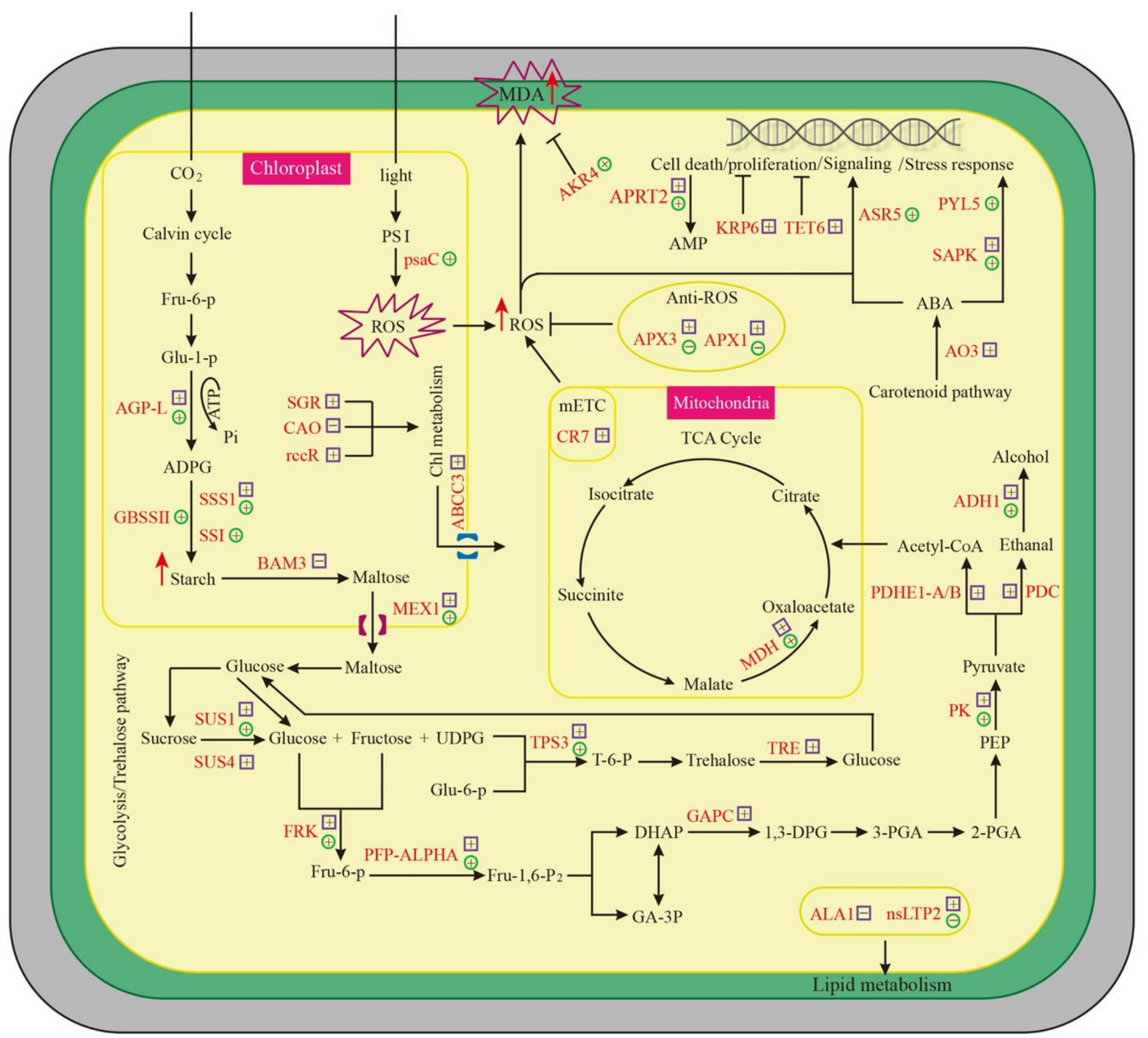

Figure 5

Schematic presentation of metabolic regulation mediated by differentially abundant genes/proteins in the Ises 1 mutant (not all the identified genes/proteins are included). The genes/proteins marked red were regulated in Ises 1 , purple square and green circle indicate transcript and protein abundance, respectively, 
"+" represents upregulation, "-" represents downregulation. AGP-L: glucose-1-phosphate adenylyltransferase large subunit; SSI: chloroplastic starch synthase; GBSSIl: chloroplastic granule-bound starch synthase Il; SSS1: chloroplastic soluble starch synthase 1; BAM3: chloroplastic $\beta$-amylase 3; MEX1: chloroplastic maltose excess protein 1-like; SUS: sucrose synthase; FRK: fructokinase-2; PFPALPHA: pyrophosphate--fructose 6-phosphate 1-phosphotransferase subunit alpha; GAPC: glyceraldehyde-3-phosphate dehydrogenase 1; PDC: pyruvate decarboxylase; PDHE1-A/B: pyruvate dehydrogenase E1 component subunit; PK: cytosolic pyruvate kinase isozyme; $A D H 1$ : alcohol dehydrogenase 1; MDH: malate dehydrogenase; TPS3: trehalose-6-phosphate synthase 3; TRE: trehalase; psaC: photosystem I iron-sulfur centre; CR7: cytochrome bc1 complex subunit; APX: L-ascorbate peroxidase; AKR4: aldo-keto reductase; SGR: chloroplastic stay-green protein; CAO: chloroplastic chlorophyllide a oxygenase; rccR: putative red chlorophyll catabolite reductase; ABCC3: ABC_transporter; ALA1: phospholipid-transporting ATPase 1; nsLTP2: non-specific lipid-transfer protein 2; AO3: putative aldehyde oxidase; APRT: adenine phosphoribosyltransferase form 2; KPR6: cyclin-dependent kinase inhibitor 6; TET6: tetraspanin-6; ASR5: abscisic stress-ripening protein 5; PYL5: ABA receptor 5; SAPK3: serine/threonine-protein kinase; ADPG: adenosine diphosphate glucose; UDPG: uridine diphosphate glucose; DHAP: dihydroxyacetone phosphate; 1,3-DPG: 1,3-diphosphoglycerate; 3-PGA: 3phosphoglycerate; 2-PGA: 2-phosphoglycerate; PEP: phosphoenolpyruvate; AMP: adenosine monophosphate. " $\rightarrow$ " indicates positive regulation, "-|" represents inhibition, and red " $\uparrow$ " indicates increased content of metabolites determined by biochemical assays.

\section{Supplementary Files}

This is a list of supplementary files associated with this preprint. Click to download.

- TableS1.xIsx

- TableS2.xIsx

- TableS3.xIsx

- TableS4.xlsx

- TableS5.xlsx

- TableS6.xIsx

- TableS7.xlsx

- TableS8.xIsx 